\title{
Phase transition in the globalization of trade
}

\author{
M. Ángeles Serrano \\ School of Informatics, Indiana University, Bloomington 47406, IN, USA \\ E-mail: mdserran@indiana.edu
}

\begin{abstract}
Globalization processes interweave economic structures at a worldwide scale, trade playing a central role as one of the elemental channels of interaction among countries. Despite the significance of such phenomena, measuring economic globalization still remains an open problem. More quantitative treatments could improve the understanding of globalization at the same time that help a formal basis for comparative economic history. In this letter, we investigate the time evolution of the statistical properties of bilateral trade imbalances between countries in the trade system. We measure their cumulative probability distribution at different moments in time to discover a sudden transition circa 1960 from a regime where the distribution was always represented by a steady characteristic function to a new state where the distribution dilates as time goes on. This suggests that the rule that was governing the statistical behavior of bilateral trade imbalances until the 60 's abruptly changed to a new form persistent in the last decades. In the new regime, the figures for the different years collapse into a universal master curve when rescaled by the corresponding global gross domestic product value. This coupling points to an increased interdependence of world economies and its onset corresponds in time with the starting of the last globalization wave.
\end{abstract}




\section{Globalization and trade}

At this point, the beginning of the third millennium in the Gregorian calendar, it seems customarily assumed that several epochs of globalization have occurred to shape the world as we perceive it today. In these periods, a complex series of changes closely intertwined develop into an increasing interdependence and interaction between people and human organizations in disparate locations of the world. These changes, when structural, are in a great part of economic nature, markets becoming natural mediators of globalization forces [1].

In terms of trade [2], there is still controversy whether the last globalization waves, the first roughly identified from 1870 till the beginning of World War I and the second from 1960 to the present, are more different than similar [3] -there is no a complete agreement either whether a third middle wave has occurred [4]. The two waves correspond to processes of decolonization and falls of technical barriers with the corresponding downloads of costs and time expenditures. The first wave was triggered by the Industrial Revolution, with steam power encouraging the expanding of railroad networks and oceanic routes and the telegraph connecting the two sides of the Atlantic. The second came intimately related to the Information Technologies Revolution, communications costs dramatically dropping at the same time that information management capabilities explode. In gross terms, the first globalization burst had to do with lower costs in transportation of materials and goods, while the second deals with exchange of information and ideas. Two different natures of changes that may well produce different impacts.

This discussion brings directly to the problem of how to measure globalization. Quantitative approaches could enlighten but have been timid to this moment, most works adopting analytical methodologies. New quantitative ways of studying globalization processes are required to exploit the wealth of information in historical data [5, 6]. One simple step forward consists in complementing the study of aggregated or global values with the statistical analysis of how they are distributed, from where we can obtain not only more detailed but also new information. For instance, a prominent figure when measuring globalization of trade plots the evolution in time of total international trade as a percentage of the global product, computed as the sum of all national gross domestic products (GDPs). As it has been reported by several authors [3, 4, 7], an U-shape pattern emerges, with rise of trade in the two eras of globalization and a major reversal in between, and has been claimed as a common trait which recurs in many other empirical analysis, such as in the plot of global capital flows to GDP ratio or in the correlation between savings and investment [3, 8].

\section{Analyzing the evolution of bilateral trade imbalances}

Still, further crucial information can be obtained from trade data if, moreover, one draws the evolution in time of the distribution of bilateral trade flows. Here we show that until 


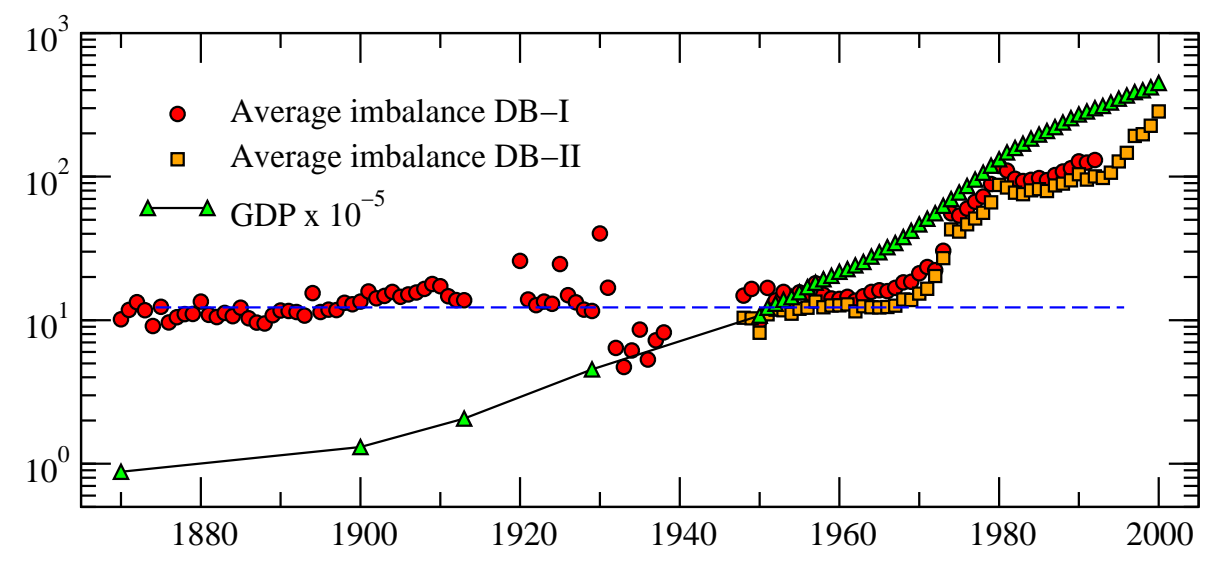

Figure 1. Historical evolution of global GDP values and average trade imbalances. From 1870 to 2000, the average value of the bilateral trade imbalances between countries in the world trade system for the two data bases that we are considering is compared to the evolution in the same period of the global gross domestic product (GDP). The results are shown on a log-linear scale for ease of comparison. The unit of measure for the average imbalance is millions of current year US dollars. GDP is also given in millions of current year US dollars, but it is rescaled to the level of bilateral trade imbalances.

1960, all the distributions overlap into a characteristic function, which afterwards evolves widening as time goes by. Most interestingly, the different trade imbalances distributions for all years since 1960 can be rescaled into a single master curve just by taking into account the evolution in time of the global GDP, which marks a characteristic scale with respect to which the system is self-similar. The breaking of the original folding at the point when a new single-curve collapse starts suggests that the rule that has been governing the statistical behavior of bilateral trade imbalances until the 60's has changed to a new scaling law in the last decades, and that this has happened in a sudden transition of the world trade system just at the beginning of the last globalization wave.

We used historical national import/export data from two different databases, DBI [9, 10] (1870-1992) and DBII [11, 12] (1948-2000). World war periods, 19141919 and 1939-1947, are avoided due to lack of reported information in the data bases. World GDP historical values are more difficult to obtain. Despite its role as a major instrument of economic policy in virtually all countries in the world, it is indeed a twentieth century concept as the rest of the national income accounts and GDP data were not collected or even defined before the 1930s. In our graphs, GDP data come from a third source [13, 14]. The bilateral trade flux $F$ between two countries is computed as the net money flow from one to the other due to trade exchanges. In Fig. 1, the time evolution of the global GDP is compared to that of the average bilateral trade flow. Both evolutions seem decoupled until the 60's. From 1870 to that date, the average imbalance remains fluctuating around a constant level, in contrast to the estimation for the global GDP, which appreciably grows. Afterwards, they couple and follow similar growing patterns. This seems indicative of a change of behavior or transition. 

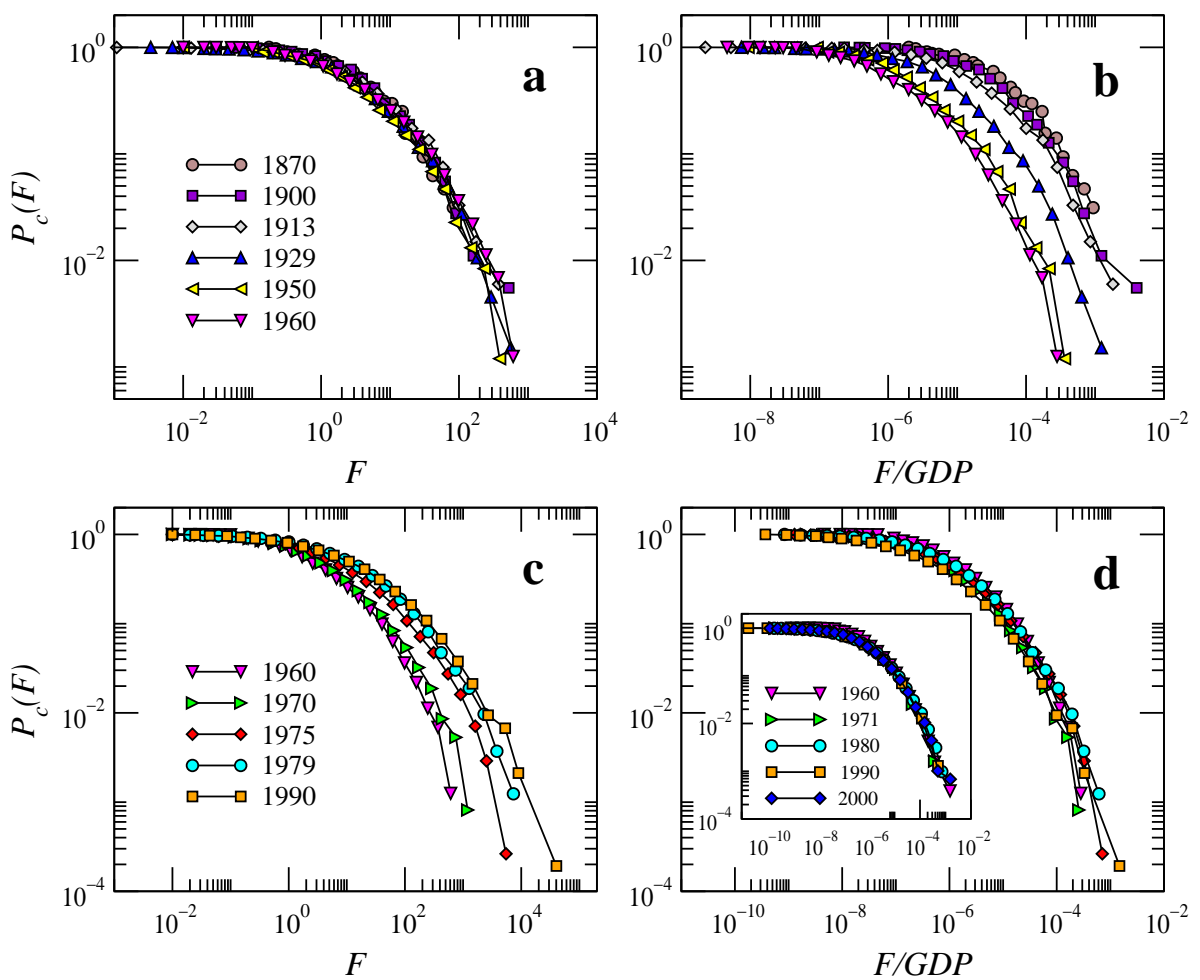

Figure 2. Master curve for the complementary cumulative probability distributions of bilateral trade imbalances. All the graphs are represented on a double logarithmic scale. The unit for all the variables is millions of current year US dollars. Complementary cumulative distributions of bilateral trade flows for several different years between 1870 and 1990 are shown in (a) and (c). Until 1960, all the distributions overlap on a characteristic curve (a). Afterwards, the distributions broaden as time goes on (c). In (b) and (d), we show the rescaling of the previous graphs. The values on the $F$ axis has been divided by the GDP value for the corresponding year. The original characteristic curve prevailing before 1960 is broken (b), while a new master curve emerges from the collapse of the data between 1960 and 1990 (d). The inset in (d) shows that this collapse is also found when analyzing data from a different source which include the year 2000 .

In Fig. 2, we present the complementary cumulative probability distribution of net trade flows between pairs of countries for several different years since 1870 . The curves are measuring the probability that a trade imbalance between two countries in the trade system is bigger than a certain amount, and the cumulative evaluation offers the advantage of filtering out the statistical noise due to the finite sizes of the samples without loosing information about the distribution. The first to observe is that, in good approximation, all the curves overlap between the years 1870 and 1960, see Fig. 2(a), moment in which the folding is broken and the distributions evolve widening year after year, see Fig. 2(c). We now rescale the curve for each year by taking into account the global GDP value in that period. For each distribution, we apply the transformation $F \longrightarrow F / G D P$ which divides the fluxes in the horizontal $\mathrm{F}$ axis by the corresponding global GDP value. Notice that this transformation would produce the same result if real 
values were used instead of nominal values. The results are shown in Fig. 2(b) and (d). In Fig. 2(b), we just corroborate that the collapse does not work for the years between 1870 to 1960, the transformation indeed breaks the original folding. On the contrary, in Fig. 2(d) all the distributions for different years since 1960 and until 2000 and for the two different databases under consideration show an excellent collapse into a single master curve. Imports and exports on their own are seen to present the same behavior, due to the high correlation between the levels of imports and exports in every single trade channel. All these distributions are lognormal -they can be thought of as the multiplicative product of many small independent factors-, again an ubiquitous shape in economics. They can be adjusted to the form $1 / 2-1 / 2 * \operatorname{erf}[(\ln (x)-\mu) /(\sigma \sqrt{2})]$, where $\mu$ and $\sigma$ are the mean and standard deviation of $\ln (x)$ and erf stands for the error function. For the characteristic curve in Fig. 2(a), $x \equiv F$ and the parameters are $\mu=1.14$ and $\sigma=1.88$. The parameters for the master curve in Fig. 2(d) with $x \equiv F / G D P$ are $\mu=-14.10$ and $\sigma=2.34$, and for the inset $\mu=-14.39$ and $\sigma=2.55$, so the two databases produce consistent information.

The collapse of the distribution for the different years into a master curve implies that the system is self-similar with respect to the characteristic scale given by the GDP. That means that the widening of the distributions in time is just a dilatation driven by the increase in total GDP and the same curve is found once the GDP growth is discounted. Until new structural changes impact the world trade system, we can assume that this behavior will be preserved through time so that, by taking into account global GDP projection values, we can predict the statistical distribution of bilateral trade imbalances that would correspond to future periods.

\section{Discussion}

To understand better how GDP and trade imbalances are related, the empirically successful gravity model of international trade can be revisited [15]. In its basic form, it predicts bilateral trade flows based on a functional form that is reminiscent of the law of gravity in physics, and involves the distance between pairs of countries and their economic masses, estimated in first instance as their GDP. Thus, bilateral trade imbalances seem to be empirically dictated in part by the GDP values of the countries concerned. On the other hand, the aggregated value of all bilateral trade flows for a certain country affects in its turn GDP levels. The GDP of a country is defined as the market value of all final goods and services produced within its borders in a given period of time. In the expenditure-based approach, it is decomposed in several terms as $G D P(t)=C(t)+I(t)+G(t)+F(t)$, where $C(t)$ stands for private consumption, $I(t)$ for business investments in capital, $G(t)$ for government spending, and $F(t)$ stands for net trade balance. So, internal contributions are corrected by the trade interactions with other countries. The sudden transition in the 60's ties, from that moment, the evolution

of the statistical distribution of net trade flows to that of the global GDP. Although not a proof, this seems to suggest that the internal components of the economies (private, 
business and government spending) become more dependent on trade exchanges with other countries, and this leads us to conjecture that GDP and international trade, or in other words internal and external components, are entangled in a complex continuous feedback mechanism. At this stage, this idea is purely speculative and further support should be provided in future work. Nevertheless, the integration of markets in the last decades does not seem to come in a smooth gradual transformation but rather in a fault transition. Whether this is indicative of the birth of a truly global market where all the economies are effectively interwoven beyond trade needs more prove. New validations about the reach of the phase transition should be performed as well. One example of technical question that immediately arises is whether the empirical success of gravity models for explaining bilateral trade as a function of GDP after the 1960 is maintained when studying historical data before this date.

In a way that is complementary to more traditional approaches in economics, we measure a sudden transition in the statistical behavior of trade imbalances which corresponds in time with the starting of the last globalization wave. From a regime where the distribution of trade imbalances was steady and independent of the evolution of GDP, a new state is reached where the global GDP marks the characteristic scale and the distribution dilates as GDP grows in time. In the new regime, the distributions for the different years collapse into a single universal master curve when rescaled by the corresponding global GDP value. Although more work should be done in order to clarify the relation between these empirical facts and the effects of the last wave of globalization in trade, the empirical findings in this paper point to an abrupt transformation that has qualitatively and quantitatively increased the interdependence of world economies through trade. From these results we can conjecture that the segregation of factors in purely internal components, just related to individual economies, versus trade, implying interactions with other countries, seems not to be neat any more. As a conclusion, the mere aggregation of bilateral trade exchanges is not enough to explain the emergent behavior of the global trade system, that, on the other hand, we can predict at the statistical level. While we do not see any other radical revolutions impacting the trade system, and maybe this is not so difficult, the self-similar character of the distribution of bilateral trade imbalances allows us to anticipate its form for the forthcoming years.

\section{Acknowledgments}

We thank Marián Boguñá and Alessandro Vespignani for helpful comments.

\section{References}

[1] Theodore Levitt. The globalization of markets. Harvard Business Review, May-June, 1983.

[2] Paul R. Krugman. Growing world trade: Causes and consequences. Brookings Papers on Economic Activity, 1995(1):327-377, 1995.

[3] Richard E. Baldwin and Philippe Martin. Two Waves of Globalisation: Superficial Similarities, Fundamental Differences, Globalization and Labor, ed. H. Siebert (Tübingen: Mohr) 3, 1999. 
[4] Christopher Chase-Dunn, Yukio Kawano, and Benjamin D. Brewer. Trade globalization since 1795: Waves of integration in the world-system. American Sociological Review, 65:77-95, 2000.

[5] Angus Maddison. The World Economy: a millenial perspective. OECD Development Centre, Paris, France, 2001.

[6] Angus Maddison. The World Economy: historical statistics. OECD Development Centre, Paris, France, 2003.

[7] A. Estevadeordal, B. Frantz, and A. M. Taylor. The rise and fall of world trade, 1860-1939. Quarterly Journal of Economics, 118:359-407, 2003.

[8] Alan M. Taylor. Globalization and new comparative economic history. NBER Reporter: Research Summary, Winter 2005/6, 2005. http://www.nber.org/reporter/winter06/taylor.html.

[9] K. Barbieri. The Liberal Illusion: Does Trade Promote Peace? University of Michigan Press, Ann Arbor, USA, 2002.

[10] Data available at http://sitemason.vanderbilt.edu/site/k5vj7G/new_page_builder_4.

[11] K. S. Gleditsch. Expanded trade and gdp data. J. Conflict Resolut., 46:712724, 2002.

[12] Data available at http://weber.ucsd.edu/ kgledits/exptradegdp.html.

[13] Angus Maddison. Contours of the World Economy: the Pace and Pattern of Change, 1-2030 AD. Cambridge University Press, 2007.

[14] Data available at http://www.ggdc.net/maddison/.

[15] Jeffrey $H$. Bergstrand. The gravity equation in international trade: some microeconomic foundations and empirical evidence. The Review of Economics and Statistics, 67(3):474-481, 1985 . 